\title{
Benefits of Enhanced Recovery After Surgery for fusion in degenerative spine surgery: impact on outcome, length of stay, and patient satisfaction
}

\author{
Bertrand Debono, MD, ${ }^{1}$ Marco V. Corniola, MD, ${ }^{2}$ Raphael Pietton, MD, ${ }^{1}$ Pascal Sabatier, MD, ${ }^{1}$ \\ Olivier Hamel, MD, PhD, ${ }^{1}$ and Enrico Tessitore, MD²
}

'Department of Neurosurgery, CAPIO-Clinique des Cèdres, Cornebarrieu, France; and 2Department of Neurosurgery, Spine Unit, Geneva University Hospitals, Geneva, Switzerland

OBJECTIVE Enhanced Recovery After Surgery (ERAS) proposes a multimodal, evidence-based approach to perioperative care. Thanks to the improvement in care protocols and the fluidity of the patient pathway, the first goal of ERAS is the improvement of surgical outcomes and patient experience, with a final impact on a reduction in the hospital length of stay (LOS). The implementation of ERAS in spinal surgery is in the early stages. The authors report on their initial experience in applying an ERAS program to several degenerative spinal fusion procedures.

METHODS The authors selected two 2-year periods: the first from before any implementation of ERAS principles (preERAS years 2012-2013) and the second corresponding to a period when the paradigm was applied widely (post-ERAS years 2016-2017). Patient groups in these periods were retrospectively compared according to three degenerative conditions requiring fusion: anterior cervical discectomy and fusion (ACDF), anterior lumbar interbody fusion (ALIF), and posterior lumbar fusion. Data were collected on patient demographics, operative and perioperative data, LOSs, 90-day readmissions, and morbidity. ERAS-trained nurses were involved to support patients at each pre-, intra-, and postoperative step with the help of a mobile application (app). A satisfaction survey was included in the app.

RESULTS The pre-ERAS group included 1563 patients (159 ALIF, 749 ACDF, and 655 posterior fusion), and the postERAS group included 1920 patients (202 ALIF, 612 ACDF, and 1106 posterior fusion). The mean LOS was significantly shorter in the post-ERAS group than in the pre-ERAS group for all three conditions. It was reduced from $6.06 \pm 1.1$ to $3.33 \pm 0.8$ days for the ALIF group $(p<0.001)$, from $3.08 \pm 0.9$ to $1.3 \pm 0.7$ days for the ACDF group $(p<0.001)$, and from $6.7 \pm 4.8$ to $4.8 \pm 2.3$ days for posterior fusion cases $(p<0.001)$. There was no significant difference in overall complications between the two periods for the ALIF (11.9\% pre-ERAS vs $11.4 \%$ post-ERAS, $p=0.86)$ and ACDF $(6.0 \%$ vs $8.2 \%, p=0.12)$ cases, but they decreased significantly for lumbar fusions $(14.8 \%$ vs $10.9 \%, p=0.02)$. Regarding satisfaction with overall care among 808 available responses, 699 patients (86.5\%) were satisfied or very satisfied, and regarding appreciation of the mobile e-health app in the perceived optimization of care management, 665 patients $(82.3 \%)$ were satisfied or very satisfied.

CONCLUSIONS The introduction of the ERAS approach at the authors' institution for spinal fusion for three studied conditions resulted in a significant decrease in LOS without causing increased postoperative complications. Patient satisfaction with overall management, upstream organization of hospitalization, and the use of e-health was high. According to the study results, which are consistent with those in other studies, the whole concept of ERAS (primarily reducing complications and pain, and then reducing LOS) seems applicable to spinal surgery.

https://thejns.org/doi/abs/10.3171/2019.1.FOCUS18669

KEYWORDS Enhanced Recovery After Surgery; fast-track surgery; spine surgery; interbody fusion; mobile app; e-health

I NTRODUCED by Henrik Kehlet in 1997, ${ }^{16}$ Enhanced Recovery After Surgery (ERAS) proposes a multimodal, evidence-based approach to perioperative care. Thanks to the overall improvement in care protocols and the fluidity of the patient pathway, the first goal of ERAS is the improvement of surgical outcomes and patient experience, with an ultimate impact on a reduction in the length of stay (LOS). ${ }^{15}$ ERAS procedures involve the optimization of each pre-, intra-, and postoperative stage, placing the patient in a central and proactive position in his or her own

ABBREVIATIONS ACDF = anterior cervical discectomy and fusion; ALIF = anterior lumbar interbody fusion; app = application; ASA = American Society of Anesthesiologists; ERAS = Enhanced Recovery After Surgery; LOS = length of stay; VAS = visual analog scale.

SUBMITTED November 30, 2018. ACCEPTED January 17, 2019.

INCLUDE WHEN CITING DOI: 10.3171/2019.1.FOCUS18669. 
management. ${ }^{20}$ ERAS programs are successfully developing in most areas of surgery and offer results that justify the buzz surrounding this concept. ${ }^{21}$ Spinal surgery was finally considered late in the process in this new multimodal organization. After the first (German) study by Fleege et al. ${ }^{10}$ demonstrating the value of preoperative patient education in spinal surgery, Wang et al. published the first consecutive series of lumbar fusion cases, detailing key points in their practice, especially the anesthesiological and technical aspects of ERAS. ${ }^{36}$ Wainwright et al..$^{34}$ proposed additional methods for the implementation of ERAS protocols in major spinal surgery. However, the bibliography of peerreviewed studies combining ERAS and spinal surgery, although promising, is still in the early stages. ${ }^{35}$ Thanks to a synergistic vision of the health group and caregivers at our institution, ${ }^{33}$ multidisciplinary work on the implementation of ERAS has been done at our institution since the end of 2013, and in the past we have reported on the application of these techniques in outpatient lumbar spine surgery. ${ }^{6,8}$ We strongly assume that the transition to outpatient surgery (recently in Europe) follows exactly the same rules as those of ERAS. Following these first clinical trials, we report on our initial experience in applying an ERAS program to several degenerative spinal fusion procedures.

\section{Methods \\ Type of Study}

This is a retrospective analysis of prospectively gathered data from our institutional registry, which contains the records of all patients who underwent spinal fusion for degenerative conditions. The local ethics committee approved the study.

\section{Definition of the Groups and Periods}

At our institution, the ERAS approach has been implemented since the end of 2013, and the multimodal changes required at each pre-, intra-, and postoperative step have been established progressively. The different types of surgeries (lumbar disc herniation, decompression, anterior cervical discectomy and fusion [ACDF], other fusions, etc.) have been gradually included in the approach to facilitate the adoption of this paradigm shift by all stakeholders, including patients.

We devoted the current study to fusion procedures and selected two 2-year periods, the first from before any implementation of ERAS principles (pre-ERAS years 2012-2013) and the second corresponding to a period when the paradigm was applied widely (post-ERAS years 2016-2017).

We included in these periods all consecutive patients scheduled for three types of elective degenerative spine surgery with fusion: anterior lumbar interbody fusion (ALIF), ACDF, and any posterior or posterolateral fusion (posterior lumbar interbody fusion [PLIF], transforaminal lumbar interbody fusion [TLIF]), except in cases of scoliosis and large deformities that have not been considered suitable for the ERAS program at the moment.

\section{ERAS Procedure}

Preadmission and Preoperative Step

At our center, a 24-hour unit is dedicated to the support of ERAS care, has trained nurses, and is a place in which a patient briefing session is held once the intervention is scheduled (Fig. 1). ${ }^{33}$ The meeting with the surgeon is immediately followed by consultations with an anesthesiologist and physiotherapist (patient preoperative education). Then an ERAS nurse explains the pre- and postoperative stages of the procedure, as well as the prescribed home medication, and describes the main scenarios that can occur early after discharge. Nurses are on call to maintain a permanent telephone link with the patient at home. Before the patient is admitted to the hospital, he or she can consult online information about his or her future treatment and register online for hospital admission to limit excessive waiting the morning of his or her admission.

\section{Hospitalization}

Admission on the day of the procedure is made at 7 AM, with prescribed modern fasting and limited premedication. Prolonged fasting is avoided because it has been proven to exert negative effects on the metabolism and musculature. Eating is allowed until 6 hours prior to surgery, and clear liquids are permitted even up to 2 hours before, especially carbohydrate supplementation (e.g., apple juice). ${ }^{21}$ The transfer to the operating room is done by walking, without a stretcher.

\section{Intraoperative Step}

Discussions on indications and surgical techniques are outside the scope of this study, but, briefly, the techniques for all conditions were performed in the same manner in both periods. Nevertheless, our surgeons preferred minimally invasive techniques, whose basic concepts, particularly muscle sparing, are synergistic with the principles of ERAS. ${ }^{36}$ All implants in lumbar posterior procedures were positioned under intraoperative 3D imaging and navigation. ALIFs were performed retroperitoneally with the support of a vascular surgeon, with the urinary catheter removed as soon as the operation was completed. The ACDFs were performed under a microscope via an anterior approach. The operators used either stand-alone polyetheretherketone (PEEK) cages or plates, according to the surgeon's preference and the patient's case. The use of drains was drastically limited, and the daily use of braces, lumbar belts, or soft/rigid cervical collars was not promoted except in exceptional cases. The patient typically spent 2 hours in the recovery room and received early advice from the rehabilitation team.

\section{Postoperative Step}

Discharge was rapidly organized in the following days after validation by the operator according to a checklist. ${ }^{5}$ Concerning pain control, the opioid-sparing multimodal approach was adopted by immediately favoring tramadol and nonsteroidal anti-inflammatory drugs in the recovery room. ${ }^{4}$ Oxycodone was provided if pain was poorly controlled by these other agents. Patient education was focused on the use of analgesics, with particular emphasis on avoiding the use of an opioid analgesic. ${ }^{28}$ Regarding the early home followup, a nurse from the ERAS team was available 24 hours a day by phone or a dedicated mobile application (app). ${ }^{6}$ 


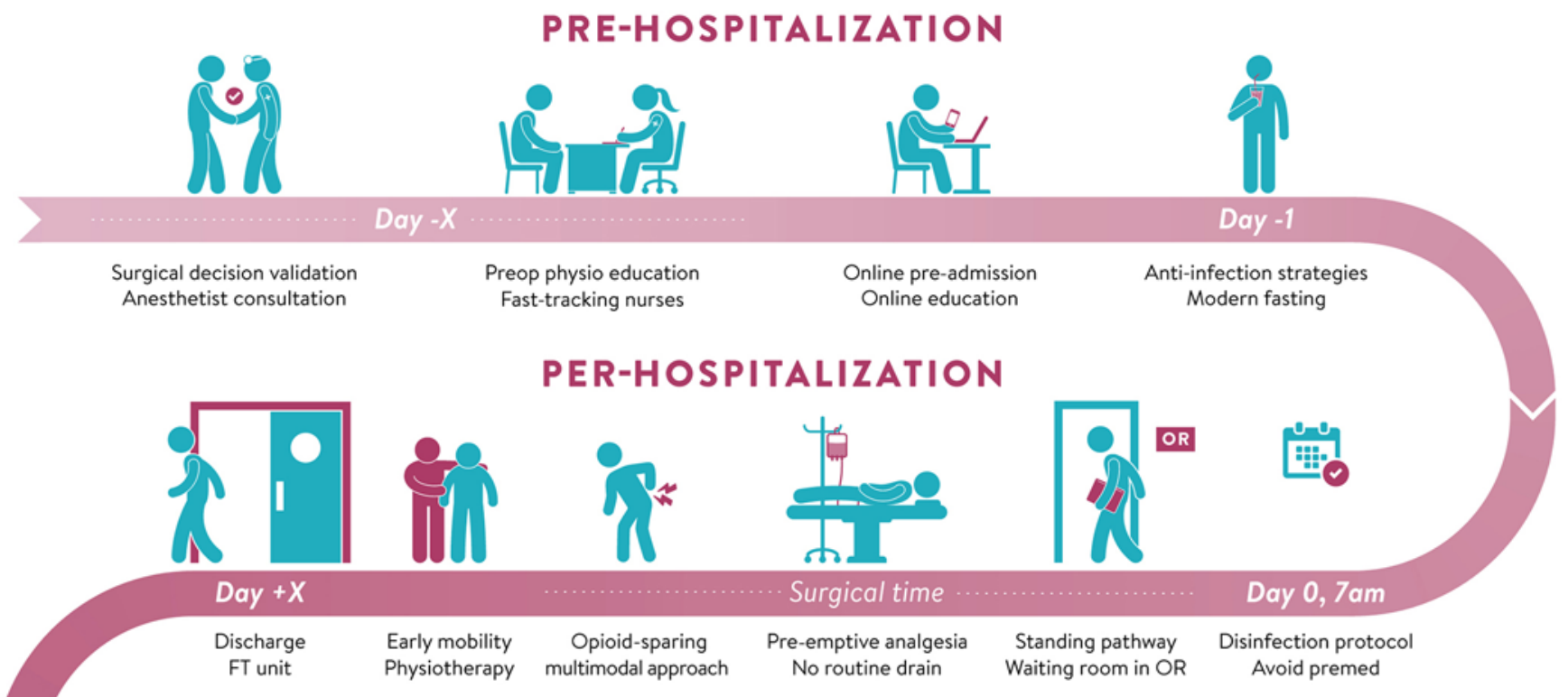

POST-HOSPITALIZATION

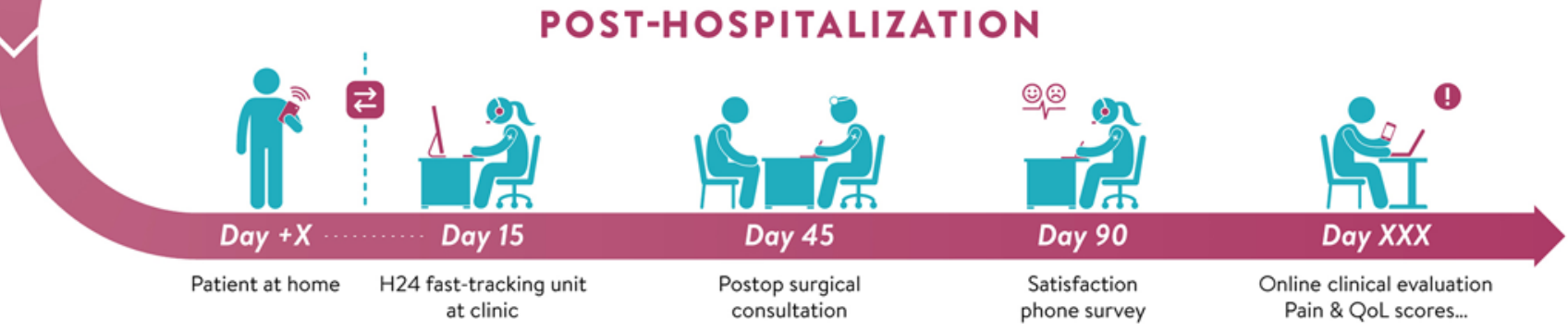

FIG. 1. Overview of ERAS procedures for spinal fusion for degenerative conditions. FT = fast-tracking (ERAS); H24 = 24-hour; OR = operating room; physio = physiotherapy; premed = premedication; $\mathrm{QoL}=$ quality of life.

\section{Postoperative Management With Dedicated Mobile App}

A dedicated mobile app (e-fitback, Nouveal e-santé) was used for the postoperative monitoring of patients in the post-ERAS group only. All consecutive patients had a smartphone, tablet, or personal computer (Fig. 2). During the preoperative consultation with ERAS nurses, a Quick Response (QR) code was given free of charge to the patient to load the app on the platform of his or her choice and for connection from 48 hours prior to surgery until the 15 th postoperative day.

The mobile app recovery indicators included a visual analog scale (VAS) for pain and a questionnaire regarding body temperature, a painful voiding disorder, a motor disorder, or a blood stain on the dressing. The patient interface has a checklist that sends an alarm to the ERAS team with different color codes based on the information entered by the patient (Fig. 2). Patients must validate the checklist at least once during the first postoperative 48 hours; otherwise a nonresponse alarm is sent to the ERAS team, and the patient is immediately contacted. The frequency of use is unlimited during the 15 days.

The available alarms include the following: nonresponse alarm, triggered if the patient does not validate a checklist within the first 48 hours; green alarm, activated if the patient has a VAS score $<6$, a fever $<38^{\circ} \mathrm{C}$, no neu- rological symptoms, or no blood stain on their dressing (this alarm does not trigger a specific reaction from the ERAS team); and emergency (red) alarm, activated if the patient has a VAS score $>6$, a fever $>38^{\circ} \mathrm{C}$, voiding difficulty, a new neurological deficit, or a blood stain on their dressing (this alarm triggers a reaction from the ERAS team, which contacts the patient quickly, 24 hours a day).

\section{Outcome Assessment}

A surgical consultation was routinely undertaken at 6 weeks postoperatively, during which neurological and pain statuses were recorded. Postoperative endpoints analyzed included death, systemic complications, wound complications (superficial wound infection, deep surgical site infection), and rehospitalizations at 90 days, which were considered as any surgical revision in this interval. ${ }^{26} \mathrm{We}$ used the classification proposed by Rampersaud et al. ${ }^{25}$ to stratify complications into two groups: Major complications were defined as events requiring significant treatment, an increased LOS by $>7$ days, long-term sequelae lasting $>6$ months, or death. Minor complications were defined as events requiring no or minimal treatment, an increased LOS by 2-7 days, and no sequelae lasting $>6$ months.

Any subsequent appointments were then based on clinical need. 


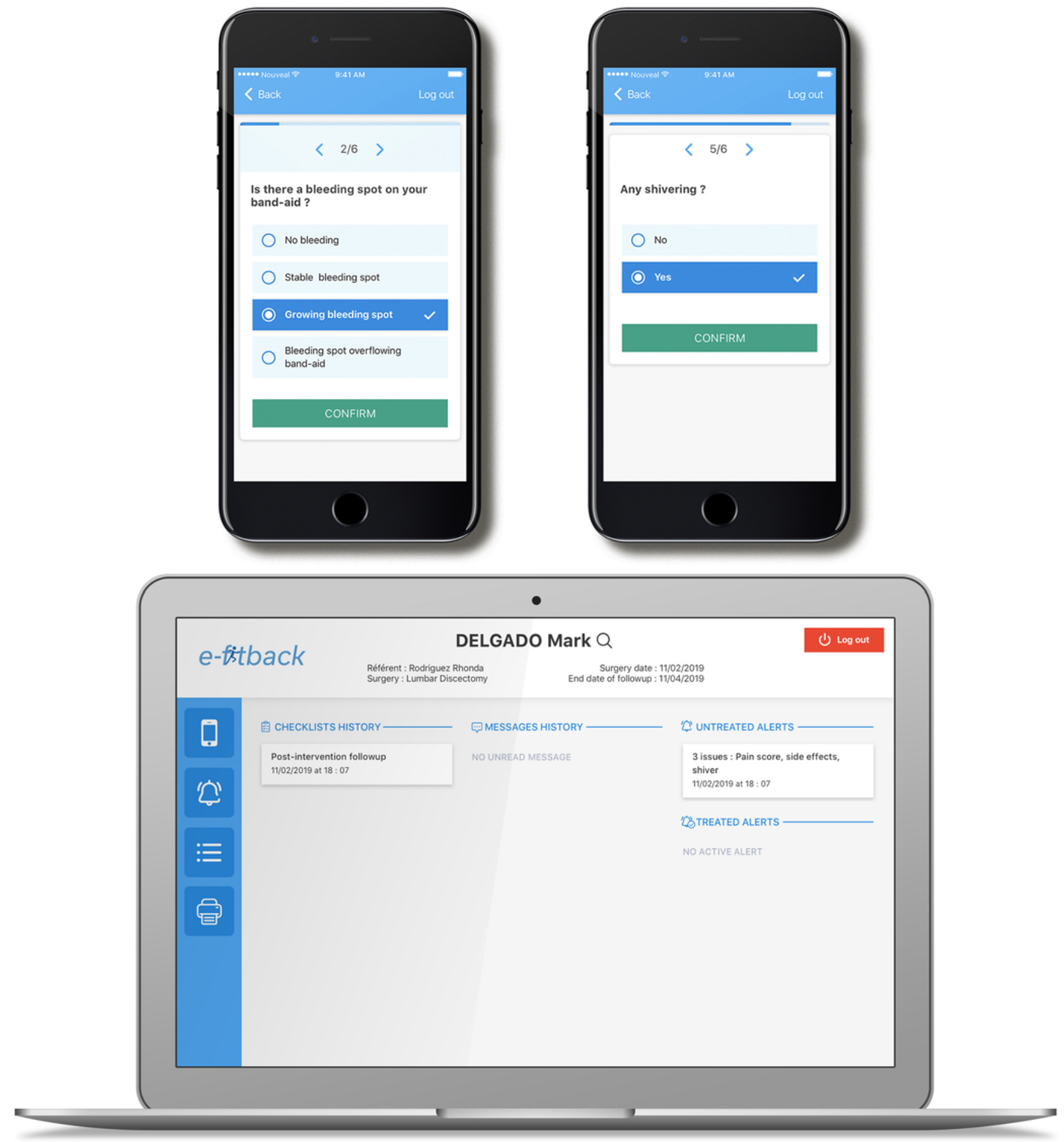

FIG. 2. Mobile app dedicated to patient home monitoring (patient and nurse interface). Copyright Nouveal e-santé. Published with permission.

\section{Satisfaction Survey}

The mobile app was also used to conduct an online survey at the end of the e-health follow-up period in the post-ERAS group only. Five-point Likert scales (from $5=$ very satisfied to $1=$ very unsatisfied) were used to assess patient satisfaction, including satisfaction with 1) overall care, 2) organization of discharge, 3) organization of stay preparation, and 4) the mobile e-health app in the perceived optimization of care management.

\section{Statistical Analysis}

Continuous variables are summarized as the mean \pm 
TABLE 1. Demographic data according to the two periods under study

\begin{tabular}{|c|c|c|c|}
\hline Variable & $\begin{array}{c}\text { Pre-ERAS } \\
(2012-2013)\end{array}$ & $\begin{array}{l}\text { Post-ERAS } \\
(2016-2017)\end{array}$ & $\begin{array}{c}p \\
\text { Value }\end{array}$ \\
\hline \multicolumn{4}{|l|}{ ALIF } \\
\hline Sample size & 159 & 202 & \\
\hline Age in yrs & $44.5 \pm 8.6$ & $46.3 \pm 10.7$ & 0.58 \\
\hline Female & $90(56.6 \%)$ & $99(49.0 \%)$ & 0.18 \\
\hline $\mathrm{BMl}$ in $\mathrm{kg} / \mathrm{m}^{2}$ & $25.2 \pm 4.2$ & $25.7 \pm 6.1$ & 0.31 \\
\hline ASA I & $95(59.7 \%)$ & $129(63.9 \%)$ & 0.49 \\
\hline Tobacco & $78(49 \%)$ & $79(39.1 \%)$ & 0.07 \\
\hline Multilevel surgery & $24(15.1 \%)$ & $36(17.8 \%)$ & 0.58 \\
\hline \multicolumn{4}{|l|}{ ACDF } \\
\hline Sample size & 749 & 612 & \\
\hline Age in yrs & $47.6 \pm 9.9$ & $48.7 \pm 8.7$ & 0.27 \\
\hline Female & $342(45.6 \%)$ & $300(49.0 \%)$ & 0.23 \\
\hline $\mathrm{BMI}$ in $\mathrm{kg} / \mathrm{m}^{2}$ & $25.3 \pm 5.1$ & $27.3 \pm 5.0$ & 0.11 \\
\hline ASA I & $427(57 \%)$ & $320(52.3 \%)$ & 0.09 \\
\hline Tobacco & $382(51.0 \%)$ & 287 (46.9\%) & 0.14 \\
\hline Multilevel surgery & $82(10.9 \%)$ & $104(17.0 \%)$ & 0.001 \\
\hline \multicolumn{4}{|l|}{ Pst lumbar fusion } \\
\hline Sample size & 655 & 1106 & \\
\hline Age in yrs & $53.8 \pm 14.3$ & $56.1 \pm 10.2$ & 0.29 \\
\hline Female & $326(49.8 \%)$ & $564(51.0 \%)$ & 0.65 \\
\hline $\mathrm{BMI}$ in $\mathrm{kg} / \mathrm{m}^{2}$ & $26.5 \pm 4.0$ & $27.3 \pm 5.8$ & 0.33 \\
\hline ASA I & $306(46.7 \%)$ & $531(48.0 \%)$ & 0.63 \\
\hline Tobacco & $321(49.0 \%)$ & $454(41.0 \%)$ & 0.001 \\
\hline Construct >2 levels & $177(27.0 \%)$ & $351(31.7 \%)$ & 0.04 \\
\hline
\end{tabular}

Pst $=$ posterior

Values expressed as the mean \pm standard deviation or as frequency (percent). Boldface type indicates statistical significance.

standard deviation or as the median (interquartile range). Categorical variables are summarized by frequencies and percentages. The mean LOS and rates of complications and revision surgeries at 90 days were compared using parametric tests (Student t-test and chi-square test, respectively). A p value $<0.05$ was considered statistically significant. The analyses were undertaken using $\mathrm{R}$ (version 13.0, The R Foundation).

\section{Results}

\section{Demographic Data}

The complete population consisted of 3483 patients. The pre-ERAS group (2012-2013) included 1563 patients (159 ALIF, 749 ACDF, and 655 posterior fusion), and the post-ERAS group (2016-2017) included 1920 patients (202 ALIF, 612 ACDF, and 1106 posterior fusion). The comparative data concerning those groups are detailed in Table 1. There was no significant difference between groups among the ALIF patients in terms of age, sex, BMI, American Society of Anesthesiologists (ASA) classification, tobacco use, and uni- or multilevel procedures. Regarding the ACDF patients, the only significant dif- ference between the period groups concerned multilevel procedures, which were more frequent in the post-ERAS period $(17.1 \%$ vs $11.1 \%, \mathrm{p}=0.001)$. Among patients with posterior fusion, there were significantly fewer smokers $(40.9 \%$ vs $49.9 \%, \mathrm{p}=0.001)$ and more multilevel procedures $(31.7 \%$ vs $26.9 \%, \mathrm{p}=0.04)$ in the post-ERAS period.

\section{ERAS Effect: Reduction in LOS}

The mean LOS was significantly shorter in the postERAS group than in the pre-ERAS group for all three conditions (Fig. 3). LOS was reduced from $6.06 \pm 1.1$ to $3.33 \pm 0.8$ days for the ALIF group, from $3.08 \pm 0.9$ to 1.3 \pm 0.7 days for the ACDF group, and from $6.7 \pm 4.8$ to 4.8 \pm 2.3 days for the posterior fusion cases (for all comparisons: $\mathrm{p}<0.001$, Student t-test).

\section{Complications}

Complications data for the three types of procedures are presented in Table 2. There was no surgery-related death within 90 days after the index intervention. For ALIF patients, there was no significant difference in the rate of overall complications between the post-ERAS and pre-ERAS periods $(11.4 \%$ vs $11.9 \%$, respectively, $\mathrm{p}=$ 0.86 ), and there was no significant difference in the rehospitalization rate $(3.0 \%$ vs $3.1 \%, \mathrm{p}=0.9)$ or revision rate $(1.5 \%$ vs $1.8 \%, \mathrm{p}=1)$ at the third month after the index procedure. Likewise, for ACDF patients, there was no significant difference in the rate of overall complications between the post-ERAS and pre-ERAS periods $(8.2 \%$ vs $6.0 \%, \mathrm{p}=0.12$ ), and there was no significant difference in the rehospitalization rate $(1.5 \%$ vs $2.1 \%, \mathrm{p}=0.36)$ or revision rate $(0.8 \%$ vs $1.3 \%, \mathrm{p}=0.51)$ at the third month after the index procedure. For patients with posterior fusion, there was no significant difference in the rehospitalization rate at the third month after the index procedure $(6.1 \%$ vs $8.1 \%, p=0.12$ ), but there was a significant decrease in overall complications in the post-ERAS period (10.9\% vs $14.8 \%, \mathrm{p}=0.02)$, as well as in the surgical revision rate at month $3(3.7 \%$ vs $6.1 \%, \mathrm{p}=0.03)$.

\section{Postoperative Management After Discharge With the Mobile App}

Mobile app data were available for 1627 patients from the post-ERAS group (131 ALIF, 490 ACDF, and 1006 posterior fusion). There were no reported technical software problems. During the 15-day period, 1420 patients $(87.3 \%)$ did not trigger an emergency alarm, including 1410 patients who had completed their initial checklist within the first 48 hours (green alarm) and 10 patients who did not and thus triggered a nonresponse alarm and were contacted by the ERAS team. The latter patients showed no clinical abnormalities related to their recent surgery; 9 forgot to use the initial checklist and 1 had a defective smartphone. In the 15-day period, a total of 3962 green alerts among all patients were triggered, $97 \%$ of which were activated between 8 AM and 8 PM and $79.5 \%$ of which were triggered within the 1st week after discharge.

However, 207 patients (12.7\%) triggered an emergency alarm, automatically provoking a phone call from the ERAS team. The ERAS team's median delay in returning 


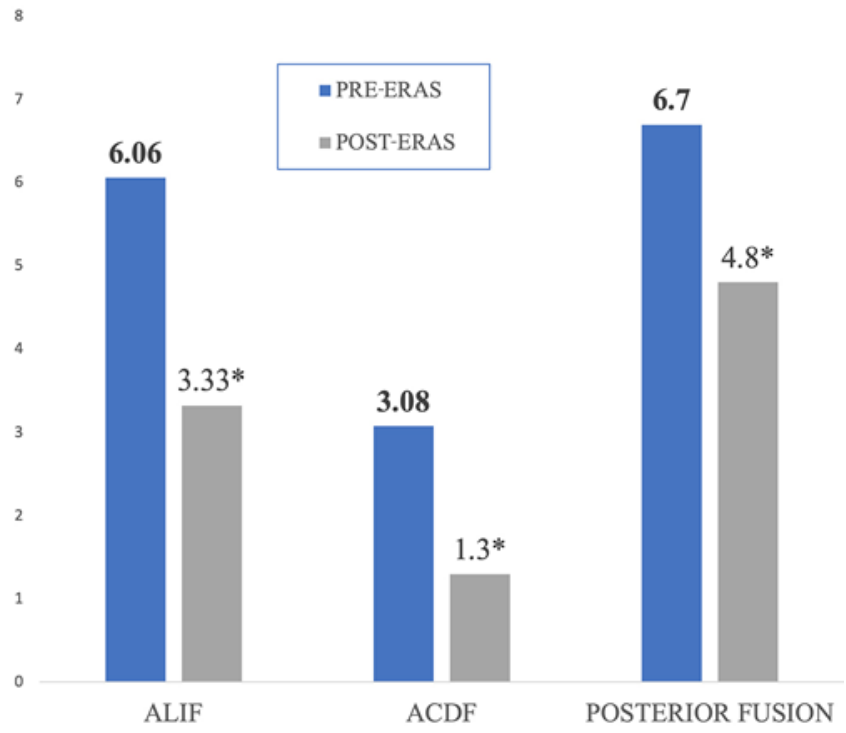

FIG. 3. Reductions in the LOS in the post-ERAS period. The y-axis represents the number of days. ${ }^{*} p<0.001$.

a patient's call was 22 minutes (range $4-47$ minutes). These 207 alarms were related to postoperative pain management and optimization of analgesics in 152 cases $(73.4 \%$ of red alarms), a low-grade fever $\left(<38.5^{\circ}\right)$ in 30 cases $(14.5 \%)$, a voiding delay in 9 cases $(4.3 \%)$, and a problem with the surgical wound or dressing in 16 cases $(7.7 \%)$.

The ERAS team managed the potential problems in 189 cases $(91.3 \%)$ by a phone briefing on the optimization of analgesics or dressing, organized an earlier consultation with the referring surgeon in 16 cases (7.7\%), and advised the patient to contact the emergency unit in 2 cases (1\%).

\section{Satisfaction Survey (Post-ERAS Group)}

At the end of the mobile app monitoring period (15 days), an online survey was offered; 808 responses could be analyzed (Fig. 4). Concerning the satisfaction with overall care, 699 patients $(86.5 \%)$ were satisfied or very satisfied. As regards the stay preparation, 750 patients (92.8\%) were satisfied or very satisfied. Regarding the organization of discharge, $671(83.0 \%)$ were satisfied or very satisfied, and concerning appreciation of the mobile e-health app in the perceived optimization of care management, 665 patients $(82.3 \%)$ were satisfied or very satisfied.

\section{Discussion}

\section{Study Findings}

The introduction of ERAS at our institution for spinal fusion in the three studied conditions has resulted in a significant decrease in LOS without causing an increase in postoperative complications, and patient satisfaction with overall management, upstream organization of hospitalization, and the use of e-health is high. We even report a significant decrease in complications in the posterior fusion group. The range of average LOS in France (2017; from https://www.atih.sante.fr/) is 4.87-6.84 days for ALIF (our post-ERAS group: $3.33 \pm 0.8$ days), 2.8-4.91
TABLE 2. Complications after fusion spine surgery according to the two periods under study

\begin{tabular}{|c|c|c|c|}
\hline \multirow[b]{2}{*}{ Variable } & \multicolumn{2}{|c|}{ No. $(\%)$} & \multirow[b]{2}{*}{$\begin{array}{c}p \\
\text { Value }\end{array}$} \\
\hline & $\begin{array}{l}\text { Pre-ERAS } \\
(2012-2013)\end{array}$ & $\begin{array}{l}\text { Post-ERAS } \\
(2016-2017)\end{array}$ & \\
\hline \multicolumn{4}{|l|}{ ALIF } \\
\hline Sample size & 159 & 202 & \\
\hline Overall complications & $19(11.9)$ & $23(11.4)$ & 0.86 \\
\hline Minor complications & $17(10.7)$ & $18(8.9)$ & \multirow{2}{*}{0.61} \\
\hline Major complications & $2(1.3)$ & $5(2.5)$ & \\
\hline Rehospitalization $<90$ days & $5(3.1)$ & $6(3.0)$ & 0.9 \\
\hline Revision surgery $<90$ days & $3(1.9)$ & $3(1.5)$ & 1 \\
\hline \multicolumn{4}{|l|}{ Type of complication } \\
\hline Medical & $7(4.4)$ & $7(3.5)$ & \\
\hline Wound & $5(3.1)$ & $7(3.5)$ & \\
\hline Neurological & $2(1.3)$ & $3(1.5)$ & \\
\hline Dural leakage & 0 & 0 & \\
\hline Implants & $1(0.6)$ & $2(1.0)$ & \\
\hline Urinary tract infection & $4(2.5)$ & $4(2.0)$ & \\
\hline \multicolumn{4}{|l|}{ ACDF } \\
\hline Sample size & 749 & 612 & \\
\hline Overall complications & $45(6.0)$ & $50(8.2)$ & 0.12 \\
\hline Minor complications & $29(3.9)$ & $31(5.1)$ & \multirow{2}{*}{0.28} \\
\hline Major complications & $16(2.1)$ & $19(3.1)$ & \\
\hline Rehospitalization $<90$ days & $16(2.1)$ & $9(1.5)$ & 0.36 \\
\hline Revision surgery $<90$ days & $10(1.3)$ & $5(0.8)$ & 0.51 \\
\hline \multicolumn{4}{|l|}{ Type of complication } \\
\hline Medical & $8(1.1)$ & $13(2.1)$ & \\
\hline Cervical approach & $20(2.7)$ & $17(2.8)$ & \\
\hline Implants & $13(1.7)$ & $17(2.8)$ & \\
\hline Infection & $4(0.5)$ & $3(0.5)$ & \\
\hline \multicolumn{4}{|l|}{ Pst lumbar fusion } \\
\hline Sample size & 655 & 1106 & \\
\hline Overall complications & $97(14.8)$ & $121(10.9)$ & 0.02 \\
\hline Minor complications & $54(8.2)$ & $77(7.0)$ & \multirow{2}{*}{0.03} \\
\hline Major complications & $43(6.6)$ & $44(4.0)$ & \\
\hline Rehospitalization $<90$ days & $53(8.1)$ & $67(6.1)$ & 0.12 \\
\hline Revision surgery $<90$ days & $40(6.1)$ & $41(3.7)$ & 0.03 \\
\hline \multicolumn{4}{|l|}{ Type of complication } \\
\hline Medical & $10(1.5)$ & $29(2.6)$ & \\
\hline Wound & $25(3.8)$ & $28(2.5)$ & \\
\hline Neurological & $15(2.3)$ & $16(1.4)$ & \\
\hline Dural leakage & $15(2.3)$ & $19(1.7)$ & \\
\hline Implants & $26(4.0)$ & $22(2.0)$ & \\
\hline Urinary tract infection & $6(0.9)$ & $7(0.6)$ & \\
\hline
\end{tabular}

Boldface type indicates statistical significance.

days for ACDF (our post-ERAS group: $1.3 \pm 0.7$ days), and 3.79-8.42 days for posterior fusion (our post-ERAS group: $4.8 \pm 2.3$ days). According to our results, which are consistent with those in other studies, the whole concept of ERAS-primarily reducing complications and pain, 


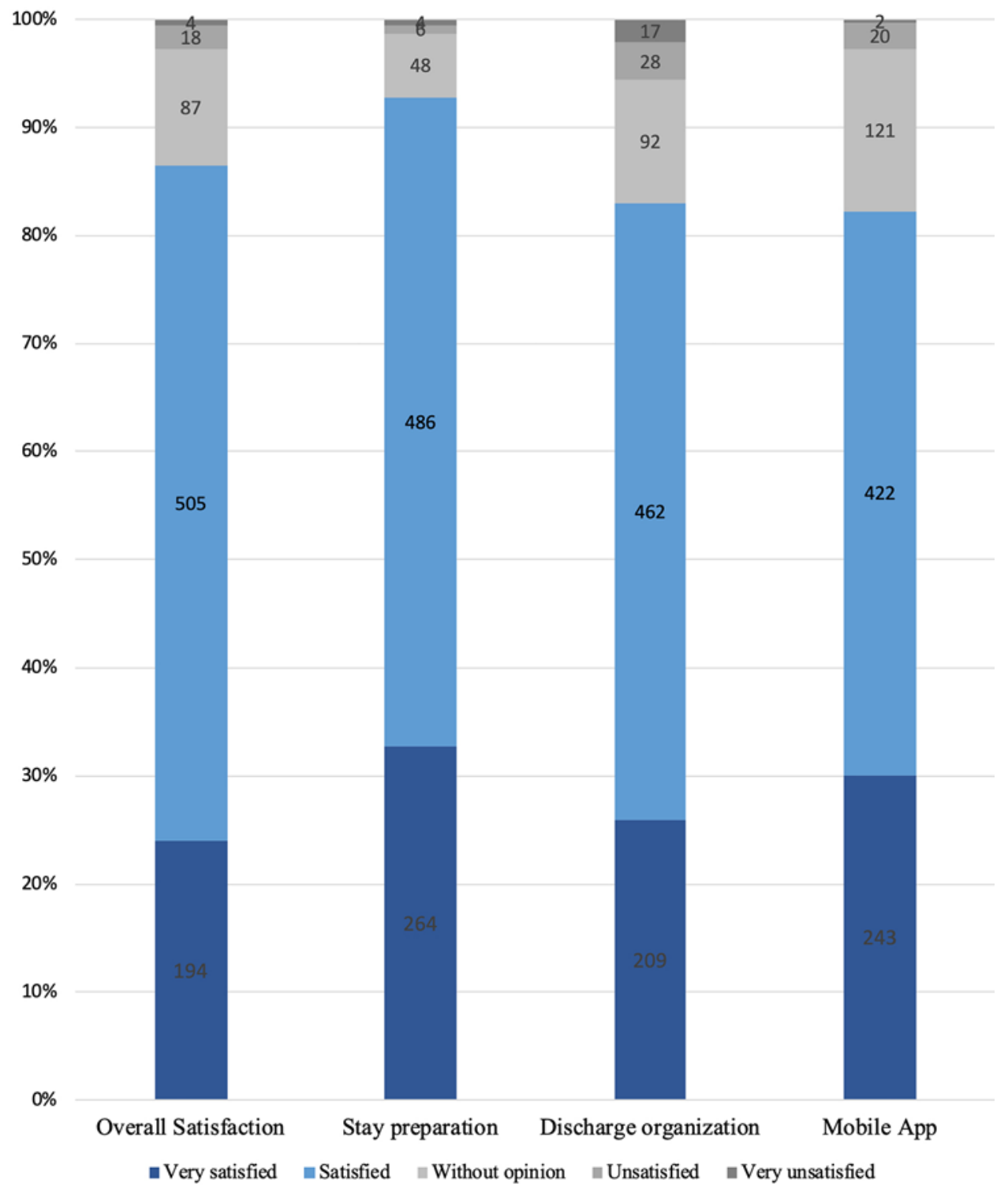

FIG. 4. Satisfaction survey conducted through a mobile app 15 days after patient discharge; 808 responses were analyzed.

and then reducing $\operatorname{LOS}^{19}$ - seems applicable to spinal surgery. ${ }^{28,36}$

\section{Specificity of Spine Surgery}

The development of ERAS protocols, first proposed for colorectoral surgery more than 20 years ago by Kehlet, extends to all surgical disciplines. ${ }^{12,18,24,29}$ The general concept remains the same: reduce morbidity and improve the patient experience, which in turn will lead to a decrease in LOS and health cost savings. The pillars of ERAS are universal: 1) a proactive patient at the center of his or her management, 2) a combination of basic evidence-based care interventions to reduce pain and enhance recovery, 3 ) a multidisciplinary change in the organizational paradigm, and 4) a data-driven iterative improvement process. ${ }^{21}$ Wang et al. proposed very innovative approaches in two seminal papers concerning ERAS and lumbar fusion without gen- eral endotracheal anesthesia. ${ }^{35,36}$ By institutional choice, however, we continue to use general anesthesia for posterior lumbar surgery, but we strongly support the authors' advocacy for a reduction in major analgesics and the expanded use of any minimally invasive technique..$^{13}$ Importantly, spinal procedures are often associated with especially high levels of pain on the first postoperative day. Lumbar fusion and complex spinal reconstruction were among the six most painful procedures in the Gerbershagen et al. ${ }^{11}$ study on pain intensity across 179 different surgical procedures. However, given the aging population, the demand for an improved quality of life, and the lack of efficacy or the exhaustion of alternative therapies, degenerative spinal fusion surgery is developing almost exponentially. ${ }^{9}$ ERAS can provide elements of optimization in a surgical field in which, unlike other specialties such as orthopedic joint surgery, spinal fusion surgery suffers from wide variabil- 


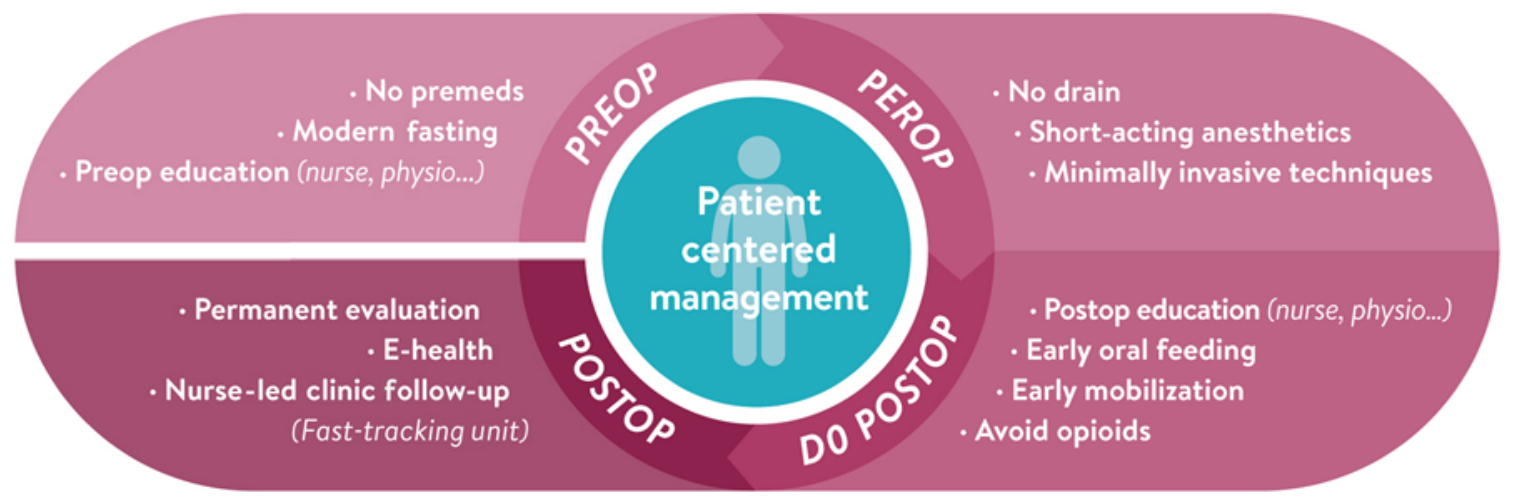

FIG. 5. ERAS, a patient-centered perioperative concept. D0 = day 0 .

ity in practitioners' indications and attitudes, as well as a high cost and very prolonged recovery. $1,7,26,34$

\section{Implementation of ERAS}

It is always difficult to fight against conservatism and resistance to the adoption of new procedures or innovations, and ERAS is no exception. ${ }^{17}$ Resistance can be found at any level and concerns all stakeholders, from the administrative levels to the healthcare staff. Basic surgical routines such as drainage, the use of a collar or brace, the timing of discharge, the use of opioids, transport home in a personal car, and so on can vary greatly and significantly influence the LOS. Upstream collegial unification of procedures and protocols of care is an essential step in the development of ERAS., ${ }^{1,33}$ Optimizing the fluidity of the patient pathway is a prerequisite, as is the appropriate postoperative follow-up. ${ }^{32}$ The entirety of care is extended and improved even if the physical stay is shortened. The administrative formalities can be settled online before the procedure to allow an expedited admission of patients and to limit undue delay. The use of e-health is very promising, allows for personalized 24-hour monitoring, and does not force postoperative problems onto the general practitioner or external emergency services. ${ }^{6,14,27,31}$ The model we propose remains at an acceptable cost to our institution, which saves money by reducing the LOS. ${ }^{8}$

\section{A Patient-Centered Approach}

One of the pillars of ERAS is to make the patient proactive (Fig. 5). Measures that could be considered anecdotal (standing patient, mobile app, etc.) force the patient to position him- or herself dynamically throughout his or her care. ${ }^{18}$ Education provided before and during hospitalization is essential to ensure the patient's safety in terms of both the functional aspect of physical activities that he or she can resume early on and the management of analgesics at home. ${ }^{3}$

\section{Involvement of All Stakeholders}

In a world in which healthcare costs are becoming a major problem regardless of the system considered, the decrease in LOS, typically associated with lower costs, becomes particularly relevant. ${ }^{2,22}$ However, the patient puts the quality and safety of care before economic savings.
Historically, public authorities and payers have pushed caregivers to reduce LOS by emphasizing the economic side of controlling healthcare spending. ${ }^{30}$ In our view, this may have created resistance among many physicians who did not want to put their patients at risk in a context of increasing medico-legal complaints. In the light of a growing scientific corpus, ERAS concepts make it possible to reverse this paradigm: Optimized protocols and high-level quality of care induce at least comparable clinical results, high patient satisfaction, and, as a second step, a decrease in LOS. $^{19}$

If safety is assured with at least comparable clinical results-in a favorable medico-economic context-ERAS will have achieved its goal. And in the end, procedures in which all stakeholders (patients, physicians, administrators, and payers) are satisfied are not very frequent in the medical field.

\section{Study Limits}

Because of the progressive adoption of ERAS at our institution, our study was retrospective. We have tried to reduce this disadvantage by using a comprehensive and prospectively maintained database including a total number of interventions that appear relevant to achieving analyzable outcomes (3483 patients). It is quite obvious that despite the promising nature of applying the ERAS approach to spinal surgery, our study does not have the rigor of a randomized controlled trial, which we know is difficult to conduct in the field of elective degenerative spine surgery. Unlike evidence-based medicine, the practice of evidence-based surgery is hampered by inherent problems and obstacles. ${ }^{23}$ At the moment, our approach does not apply to major surgeries (e.g., scoliosis, complex deformities) because of organizational issues. This is a work in progress, and other teams have started publishing on this subject. ${ }^{34}$ Finally, our study does not include an economic component. ${ }^{35}$ More detailed works will have to, on the one hand, establish the ERAS guidelines that do not yet exist for our discipline and, on the other hand, develop the medico-economic results.

\section{Conclusions}

Our study highlights the interest in applying ERAS protocols to fusion surgery for the degenerative spine. Our 
initial hypothesis that a combination of basic evidencebased care interventions to enhance recovery will subsequently decrease morbidity and the need for hospitalization in the three spine conditions we have described seems to be confirmed. The central position of the patient is a key point in this paradigm shift, which affects all stakeholders in the healthcare chain. The literature is growing, with studies demonstrating the value of the fundamental pillars of ERAS, such as patient education, multimodal pain management, strategies to minimize blood loss, and early mobilization. Obviously, any analysis of ERAS results in spine surgery must take into account the specific degenerative pathology according to the chronicity of the painful condition before the operation, its affect on the functional quality of life, and the complexity and variability of the techniques used. The corpus of publications concerning ERAS and spine surgery, still in its infancy, is beginning to grow, which should make it possible to develop guidelines for the broad implementation of these protocols that are beneficial for patients, caregivers, institutions, and payers.

\section{Acknowledgments}

We are grateful to Clarisse Paget for the processing of e-health data, Valérie Garnault, MD, from the Medical Information Department, and Jeremy Viallard for the infographics. We also thank the ERAS nurses and Valérie So, nursing staff, for their valuable contributions.

\section{References}

1. Ali ZS, Ma TS, Ozturk AK, Malhotra NR, Schuster JM, Marcotte PJ, et al: Pre-optimization of spinal surgery patients: development of a neurosurgical enhanced recovery after surgery (ERAS) protocol. Clin Neurol Neurosurg 164:142-153, 2018

2. Alvin MD, Miller JA, Lubelski D, Rosenbaum BP, Abdullah $\mathrm{KG}$, Whitmore RG, et al: Variations in cost calculations in spine surgery cost-effectiveness research. Neurosurg Focus 36(6):E1, 2014

3. Bradywood A, Farrokhi F, Williams B, Kowalczyk M, Blackmore CC: Reduction of inpatient hospital length of stay in lumbar fusion patients with implementation of an evidencebased clinical care pathway. Spine (Phila Pa 1976) 42:169_ 176, 2017

4. Cannesson M, Ani F, Mythen MM, Kain Z: Anaesthesiology and perioperative medicine around the world: different names, same goals. Br J Anaesth 114:8-9, 2015

5. Chung F, Chan VW, Ong D: A post-anesthetic discharge scoring system for home readiness after ambulatory surgery. J Clin Anesth 7:500-506, 1995

6. Debono B, Bousquet P, Sabatier P, Plas JY, Lescure JP, Hamel O: Postoperative monitoring with a mobile application after ambulatory lumbar discectomy: an effective tool for spine surgeons. Eur Spine J 25:3536-3542, 2016

7. Debono B, Lonjon G, Galovich LA, Kerever S, Guiot B, Eicker SO, et al: Indication variability in degenerative lumbar spine surgery: a four-nation survey. Spine (Phila Pa 1976) 43:185-192, 2018

8. Debono B, Sabatier P, Garnault V, Hamel O, Bousquet P, Lescure JP, et al: Outpatient lumbar microdiscectomy in France: from an economic imperative to a clinical standard-an observational study of 201 cases. World Neurosurg 106:891-897, 2017

9. Deyo RA: Fusion surgery for lumbar degenerative disc dis- ease: still more questions than answers. Spine J 15:272-274, 2015

10. Fleege C, Arabmotlagh M, Almajali A, Rauschmann M: [Pre- and postoperative fast-track treatment concepts in spinal surgery: patient information and patient cooperation.] Orthopade 43:1062-1064, 1066-1069, 2014 (Ger)

11. Gerbershagen HJ, Aduckathil S, van Wijck AJM, Peelen LM, Kalkman CJ, Meissner W: Pain intensity on the first day after surgery: a prospective cohort study comparing 179 surgical procedures. Anesthesiology 118:934-944, 2013

12. Gralla O, Haas F, Knoll N, Hadzidiakos D, Tullmann M, Romer A, et al: Fast-track surgery in laparoscopic radical prostatectomy: basic principles. World J Urol 25:185-191, 2007

13. Grasu RM, Cata JP, Dang AQ, Tatsui CE, Rhines LD, Hagan $\mathrm{KB}$, et al: Implementation of an Enhanced Recovery After Spine Surgery program at a large cancer center: a preliminary analysis. J Neurosurg Spine 29:588-598, 2018

14. Gunter R, Fernandes-Taylor S, Mahnke A, Awoyinka L, Schroeder C, Wiseman J, et al: Evaluating patient usability of an image-based mobile health platform for postoperative wound monitoring. JMIR Mhealth Uhealth 4:e113, 2016

15. Jones EL, Wainwright TW, Foster JD, Smith JRA, Middleton RG, Francis NK: A systematic review of patient reported outcomes and patient experience in enhanced recovery after orthopaedic surgery. Ann R Coll Surg Engl 96:89-94, 2014

16. Kehlet H: Multimodal approach to control postoperative pathophysiology and rehabilitation. Br J Anaesth 78:606617, 1997

17. Kehlet H, Delaney CP, Hill AG: Perioperative medicine-the second round will need a change of tactics. Br J Anaesth 115:13-14, 2015

18. Kehlet H, Dahl JB: Anaesthesia, surgery, and challenges in postoperative recovery. Lancet 362:1921-1928, 2003

19. Kehlet H, Thienpont E: Fast-track knee arthroplasty-status and future challenges. Knee 20 (Suppl 1):S29-S33, 2013

20. Kehlet H, Wilmore DW: Evidence-based surgical care and the evolution of fast-track surgery. Ann Surg 248:189-198, 2008

21. Ljungqvist O, Scott M, Fearon KC: Enhanced Recovery After Surgery: a review. JAMA Surg 152:292-298, 2017

22. Lubelski D, Mihalovich KE, Skelly AC, Fehlings MG, Harrop JS, Mummaneni PV, et al: Is minimal access spine surgery more cost-effective than conventional spine surgery? Spine (Phila Pa 1976) 39 (22 Suppl 1):S65-S74, 2014

23. Meshikhes AWN: Evidence-based surgery: the obstacles and solutions. Int J Surg 18:159-162, 2015

24. Murphy MA, Richards T, Atkinson C, Perkins J, Hands LJ: Fast track open aortic surgery: reduced post operative stay with a goal directed pathway. Eur J Vasc Endovasc Surg 34:274-278, 2007

25. Rampersaud YR, Moro ERP, Neary MA, White K, Lewis SJ, Massicotte EM, et al: Intraoperative adverse events and related postoperative complications in spine surgery: implications for enhancing patient safety founded on evidence-based protocols. Spine (Phila Pa 1976) 31:1503-1510, 2006

26. Rumalla K, Smith KA, Arnold PM: National rates, causes, risk factors, and outcomes associated with 30-day and 90day readmissions following degenerative posterior cervical spine surgery utilizing the Nationwide Readmissions Database. Neurosurgery 81:740-751, 2017

27. Semple JL, Sharpe S, Murnaghan ML, Theodoropoulos J, Metcalfe KA: Using a mobile app for monitoring postoperative quality of recovery of patients at home: a feasibility study. JMIR Mhealth Uhealth 3:e18, 2015

28. Soffin EM, Vaishnav AS, Wetmore D, Barber L, Hill P, Gang $\mathrm{CH}$, et al: Design and implementation of an Enhanced Recovery After Surgery (ERAS) program for minimally invasive lumbar decompression spine surgery: initial experience. Spine (Phila Pa 1976) [epub ahead of print], 2018 
29. Specht K, Kjaersgaard-Andersen P, Kehlet H, Wedderkopp N, Pedersen BD: High patient satisfaction in 445 patients who underwent fast-track hip or knee replacement. Acta Orthop 86:702-707, 2015

30. Stowers MDJ, Lemanu DP, Hill AG: Health economics in Enhanced Recovery After Surgery programs. Can J Anaesth 62:219-230, 2015

31. van der Meij E, Huirne JA, Bouwsma EV, van Dongen JM, Terwee CB, van de Ven PM, et al: Substitution of usual perioperative care by ehealth to enhance postoperative recovery in patients undergoing general surgical or gynecological procedures: study protocol of a randomized controlled trial. JMIR Res Protoc 5:e245, 2016

32. Venkata HK, van Dellen JR: A perspective on the use of an enhanced recovery program in open, non-instrumented day surgery for degenerative lumbar and cervical spinal conditions. J Neurosurg Sci 62:245-254, 2018

33. Verrier JF, Paget C, Perlier F, Demesmay F: How to introduce a program of Enhanced Recovery after Surgery? The experience of the CAPIO group. J Visc Surg 153 (6S):S33-S39, 2016

34. Wainwright TW, Immins T, Middleton RG: Enhanced recovery after surgery (ERAS) and its applicability for major spine surgery. Best Pract Res Clin Anaesthesiol 30:91-102, 2016

35. Wang MY, Chang HK, Grossman J: Reduced acute care costs with the ERAS ${ }^{\circledR}$ minimally invasive transforaminal lumbar interbody fusion compared with conventional minimally invasive transforaminal lumbar interbody fusion. Neurosurgery 83:827-834, 2018
36. Wang MY, Chang PY, Grossman J: Development of an Enhanced Recovery After Surgery (ERAS) approach for lumbar spinal fusion. J Neurosurg Spine 26:411-418, 2017

\section{Disclosures}

Dr. Tessitore has received training fees from Spineart, DePuy Synthes, NuVasive, and Brainlab.

\section{Author Contributions}

Conception and design: Debono, Corniola, Hamel. Acquisition of data: Debono, Pietton. Analysis and interpretation of data: Debono. Drafting the article: Debono. Critically revising the article: Corniola, Pietton, Sabatier, Hamel, Tessitore. Reviewed submitted version of manuscript: Debono, Sabatier, Hamel, Tessitore. Approved the final version of the manuscript on behalf of all authors: Debono. Statistical analysis: Debono, Pietton. Administrative/technical/material support: Tessitore. Study supervision: Debono, Hamel, Tessitore.

\section{Correspondence}

Bertrand Debono: Neuroscience Pole Capio-Clinique des Cèdres, Cornebarrieu, France. bdebono@gmail.com. 